\title{
Con: should asymptomatic patients with moderate-to-severe OSA be treated?
}

The population prevalence of obstructive sleep apnoea (OSA) has increased significantly in the past 20-30 years paralleling trends in population ageing and increasing rates of overweight and obesity. A recent community-based study estimated that $84 \%$ of men and $61 \%$ of women have an apnoeahypopnoea index (AHI) of $>5$ events $\cdot h^{-1}$ of sleep, with moderate-to-severe OSA ( $\mathrm{AHI}>15$ events $\cdot \mathrm{h}^{-1}$ ) occurring in approximately $50 \%$ of men and $23 \%$ of women [1]. The most widely recognised manifestation of OSA, and the main target for treatment, is excessive daytime sleepiness. However, the majority of individuals with OSA in the general community [2] and even in sleep clinics [3] do not exhibit excessive sleepiness. This raises the important question of whether such patients should be treated.

Sleep professionals have tended to define the OSA syndrome quite broadly. For example, the international classification of sleep disorders (ICSD-3) defines OSA as: 1 ) an $\mathrm{AHI}>5$ events $\cdot \mathrm{h}^{-1}$ and one or more symptoms (i.e. sleepiness, fatigue, insomnia, snoring, subjective nocturnal respiratory disturbance or observed apnoea) or an associated medical or psychiatric disorder (i.e. hypertension, coronary artery disease, atrial fibrillation, congestive heart failure, stroke, diabetes, cognitive dysfunction or mood disorder); or 2 ) an $\mathrm{AHI} \geq 15$ events $\cdot \mathrm{h}^{-1}$ without symptoms or associated conditions. The implication behind this definition is that all such patients stand to benefit from OSA treatment since OSA is driving these symptoms and conditions. However, the evidence does not justify such an approach and if implemented it would probably be over inclusive and have major implications for health service delivery worldwide. For example, applying this ICSD-3 definition to data from two recent community-based cohort studies showed that $50-70 \%$ of the study populations had this OSA "syndrome" [4, 5].

Mild OSA (AHI 5-15 events $\cdot \mathrm{h}^{-1}$ ) appears to have minimal associated morbidity and response to treatment is marginal at best [6]. After reviewing the evidence, UK and Canadian technology assessments have not supported the use of CPAP for such patients $[7,8]$. Second, it must be questioned whether all patients with $\mathrm{AHI}>15$ events $\cdot \mathrm{h}^{-1}$ should be treated. Estimates from recent population studies are that up to $50 \%$ have an $\mathrm{AHI} \geq 15$ events $\cdot \mathrm{h}^{-1}$, only $2-7 \%$ of whom complain of sleepiness (Epworth sleepiness scale $($ ESS) $>10)[4,5]$.

Randomised controlled trials convincingly show that CPAP treatment of patients with moderateto-severe OSA improves sleepiness, with probable concomitant improvements in sleepiness-related quality of life and attention-dependent cognitions. There is a strong rationale therefore for a trial of CPAP (or mandibular advancement splint) in patients with moderate-to-severe OSA who are sleepy with the expectation of symptomatic improvement and reduction in accident risk. Although, it is worth remembering that sleepiness in the general population is more strongly associated with

@ERSpublications

In a large proportion of patients with moderate-severe OSA who do not report daytime sleepiness there is no convincing evidence at present that CPAP or similar treatments provide benefit for associated medical disorders, such as hypertension and diabetes http://ow.ly/qqqi30nok4d

Cite as: Vakulin $A$ Chai-Coetzer CL, McEvoy RD Con: should asymptomatic patients with moderateto-severe OSA be treated? Breathe 2019; 15: 11-14. 
conditions such as diabetes, obesity, depression and chronic sleep restriction than with OSA [9], which may in part explain why $30-40 \%$ of OSA patients with excessive sleepiness continue to feel sleepy even after optimal treatment [10].

What then of the many people in the community and in clinics who have moderateto-severe OSA without daytime sleepiness? OSA has been associated in cohort studies with several significant adverse health effects including hypertension, increased cardiovascular morbidity and mortality, depression, cognitive dysfunction, and diabetes. Should we be advocating for screening programmes to identify and treat those with moderate-to-severe OSA regardless of sleepiness, in the expectation that OSA treatment will also treat or at least ameliorate these associated diseases and disorders? Will OSA treatment benefit non-sleepy individuals with moderate-to-severe OSA ( $\mathrm{AHI} \geq 15$ events $\left.\cdot \mathrm{h}^{-1}\right)$ ?

\section{Blood pressure and hypertension}

Meta-analyses of cohort studies have found that moderate-to-severe OSA is associated with incident hypertension [11]. There is also a significant body of evidence from clinical trials that CPAP treatment produces a fall, albeit small $(2-4 \mathrm{mmHg})$, in blood pressure [12]. However, there is no randomised controlled trial evidence to show that treatment of OSA prevents the onset of hypertension or treats hypertension (i.e. can be used to replace or reduce dependence on anti-hypertensive medication) [13] Moreover, a cross-over comparative effectiveness study in newly diagnosed OSA patients with untreated hypertension showed that a standard dose of an angiotensin receptor antagonist resulted in a 4-5-fold greater reduction in 24-h blood pressure than CPAP [14]. There is also growing evidence that CPAP treatment of OSA has littleto-no effect on blood pressure in the absence of daytime sleepiness $[15,16]$. A recent meta-analysis by BRATTON et al. [16] focused on the effect of CPAP therapy on blood pressure in minimally symptomatic OSA patients. Four randomised controlled trials ( $n=1206$ patients) were included in the metaanalysis: average ESS score of 7.0 and mean \pm SD baseline diastolic and systolic blood pressure $131.2 \pm 15.8$ and $80.9 \pm 10.4 \mathrm{mmHg}$, respectively. No significant changes in either systolic or diastolic blood pressure were found during follow-up, which ranged between 1 month and 4 years. Another more recent meta-analysis by ZHANG et al. [15] included three additional trials to the report by BRATTON et al. [16] ( $n=1541$ patients almost all of whom had ESS $\leq 10)$. The findings were generally consistent with those from the earlier meta-analysis and showed no significant benefit of CPAP therapy on systolic blood pressure, and only a minor clinically insignificant decrease in diastolic blood pressure $(-0.92 \mathrm{mmHg})$.

\section{Cardiovascular events}

SAVE, the largest randomised secondary prevention trial in individuals with moderate-to-severe OSA found no benefit of CPAP treatment on major cardiovascular events [17]. A meta-analysis combining these data with several other recent randomised studies that reported on cardiovascular outcomes found a similar null effect [18]. SAVE study participants had moderate-to-severe OSA and most patients were asymptomatic or minimally symptomatic (mean \pm SD ESS in the active and control arms $7.3 \pm 3.6$ and $7.5 \pm 3.6$, respectively).

\section{Diabetes}

Cohort studies have reported an independent association between OSA and incident type 2 diabetes [19]. While proof-of-concept studies using 1-2 weeks of supervised, in-patient, 8 -h per night CPAP treatment have shown improved glycaemia control in small numbers of OSA patients with type 2 diabetes mellitus, a recent meta-analysis of randomised controlled trials of CPAP treatment $(n=581)$ found no change in glycaemic control [20].

\section{Mood disorders}

A 2015 systematic review and meta-analysis of trials examining the effects of CPAP therapy for OSA on mood reported a reduction in depressive symptoms using standardised questionnaires in pre-post treatment studies [21]. Small-to-moderate effect size improvements in depression and anxiety were also found for five randomised controlled trials of CPAP versus oral placebo ( $n=219$ patients), but no change in anxiety or depression was found in four other studies $(n=169)$ that compared CPAP with sham CPAP. The reviewers considered the risk of bias of the randomised studies to be high and the overall study quality to be low. The SAVE study $(n=2717)$ also found an improvement in depression symptoms, and to a lesser extent anxiety, in CPAPtreated versus usual care groups. Self-reported daytime sleepiness also decreased in CPAPtreated SAVE participants, despite baseline mean ESS values being within the normal range [17]. These findings raise the possibility that CPAP may improve mood, particularly depression. However, as highlighted by a recent study [22], most of the screening questionnaires used in these studies incorporate questions on sleep disturbance and/ or daytime fatigue, questions that might relate to CPAP-induced improvements in sleep disturbance rather than depression and anxiety per se. Further randomised studies are needed to determine whether CPAP treatment can prevent or treat clinical depression, or at least improve anhedonia symptoms that may be more indicative of true depression in this clinical setting. 


\section{Cognitive impairment and dementia}

A recent review and meta-analysis concluded that OSA is associated with cognitive impairment across multiple domains [23]. There is, however, a relative dearth of adequately powered randomised controlled trials examining the effect of CPAP and other therapies on cognitive function. The few studies that are available have found improved attention without changes in other domains of cognition (e.g. memory and executive function) [24]. There is emerging evidence that OSA is associated with age-related cognitive decline and dementia, and very early evidence from smallscale studies suggesting that CPAP therapy might reduce markers of dementia (e.g. $\beta$-amyloid 42 , tau proteins and lactate levels) as well as slowing the progression of cognitive decline [25, 26]. However, this line of investigation is in its infancy, lacks randomised controlled trial evidence and will require a great deal more research before meaningful conclusions can be drawn.

\section{Conclusion}

The ever-increasing expansion and cost of new medical technologies and treatments is leading to significant pressures on healthcare resources worldwide. It is critically important therefore that the allocation of resources to diagnosis and treatment of any health disorder or disease be based not only on the knowledge that the disorder causes significant ill health, but also that a high level of evidence exists showing that treatments offered are effective, and cost-effective in reducing morbidity and/or mortality, and are safe. Randomised controlled trials remain the highest level of evidence for demonstrating treatment efficacy and effectiveness. To rely on lower levels of evidence can be misleading and sometimes dangerous, as shown in the example of adaptive servo ventilation treatment for central sleep apnoea in patients with heart failure. In the case of OSA, there is a firm basis for offering treatment for symptom control, most notably to reduce daytime sleepiness, with the expectation that this will improve quality of life and patient safety. However, for the large proportion of patients with moderate-to-severe OSA who do not report daytime sleepiness there is no convincing evidence at present that CPAP or similar treatments provide benefit for associated medical disorders such as hypertension and diabetes over and above those provided by existing therapies. It is possible that new methods to improve acceptance and adherence to CPAP, or entirely new, better-tolerated and more effective therapies will emerge in the future that will be shown to ameliorate these associated conditions. However, the evidence at this time is insufficient to recommend CPAP or other existing therapies for asymptomatic moderate-to-severe OSA.

\title{
Affiliations
}

\author{
Andrew Vakulin ${ }^{1,2}$, Ching Li Chai-Coetzer ${ }^{1,3}$, R. Doug McEvoy ${ }^{1,3}$ \\ ${ }^{1}$ Adelaide Institute for Sleep Health: A Flinders Centre of Research Excellence, College of Medicine and Public \\ Health, Flinders University, Adelaide, Australia. ${ }^{2}$ The NHMRC Centre of Research Excellence, NEUROSLEEP, \\ Woolcock Institute of Medical Research, The University of Sydney, Sydney, Australia. ${ }^{3}$ Respiratory and Sleep \\ Service, Southern Adelaide Local Health Network, Adelaide, Australia.
}

\section{Conflict of interest}

None declared.

\section{References}

1. Heinzer R, Vat S, Marques-Vidal P, et al. Prevalence of sleepdisordered breathing in the general population: the HypnoLaus study. Lancet Respir Med 2015; 3: 310-318.

2. Gottlieb DJ, Whitney CW, Bonekat WH, et al. Relation of sleepiness to respiratory disturbance index: the Sleep Heart Health Study. Am J Respir Crit Care Med 1999; 159: 502-507.

3. Roure N, Gomez S, Mediano O, et al. Daytime sleepiness and polysomnography in obstructive sleep apnea patients. Sleep Med 2008; 9: 727-731.

4. Adams R, Appleton S, Taylor A, et al. Are the ICSD-3 criteria for sleep apnoea syndrome too inclusive? Lancet Respir Med 2016; 4: e19-e20.

5. Heinzer R, Marti-Soler H, Haba-Rubio J. Prevalence of sleep apnoea syndrome in the middle to old age general population. Lancet Respir Med 2016; 4: e5-e6.
6. Marshall NS, Barnes M, Travier N, et al. Continuous positive airway pressure reduces daytime sleepiness in mild to moderate obstructive sleep apnoea: a meta-analysis. Thorax 2006; 61: 430-434.

7. National Institute for Health and Care Excellence. Continuous positive airway pressure for the treatment of obstructive sleep apnoea/hypopnoea syndrome. Technology appraisal guidance [TA139]. www.nice.org.uk/guidance/ta139 Date last updated: March 26, 2008.

8. CADTH. Interventions for the treatment of obstructive sleep apnea in adults: recommendations. Ottawa, CADTH, 2017. https://cadth.ca/sites/default/files/pdf/OP0525_OSA_ Treatment_Recs_Report.pdf

9. Bixler EO, Vgontzas AN, Lin HM, et al. Excessive daytime sleepiness in a general population sample: the role of sleep 
apnea, age, obesity, diabetes, and depression. J Clin Endocrinol Metab 2005; 90: 4510-4515.

10. Antic NA, Catcheside P, Buchan C, et al. The effect of CPAP in normalizing daytime sleepiness, quality of life, and neurocognitive function in patients with moderate to severe OSA. Sleep 2011; 34: 111-119.

11. Xia W, Huang Y, Peng B, et al. Relationship between obstructive sleep apnoea syndrome and essential hypertension: a dose-response meta-analysis. Sleep Med 2018; 47: 11-18.

12. Feldstein CA. Blood pressure effects of CPAP in nonresistant and resistant hypertension associated with OSA: a systematic review of randomized clinical trials. Clin Exp Hypertens 2016; 38: 337-346.

13. Barbe F, Duran-Cantolla J, Sanchez-de-la-Torre M, et al. Effect of continuous positive airway pressure on the incidence of hypertension and cardiovascular events in nonsleepy patients with obstructive sleep apnea: a randomized controlled trial. JAMA 2012; 307: 2161-2168

14. Pepin JL, Tamisier R, Barone-Rochette G, et al. Comparison of continuous positive airway pressure and valsartan in hypertensive patients with sleep apnea. Am J Respir Crit Care Med 2010; 182: 954-960.

15. Zhang D, Luo J, Qiao Y, et al. Continuous positive airway pressure therapy in non-sleepy patients with obstructive sleep apnea: results of a meta-analysis. J Thorac Dis 2016; 8 : 2738-2747

16. Bratton DJ, Stradling JR, Barbe F, et al. Effect of CPAP on blood pressure in patients with minimally symptomatic obstructive sleep apnoea: a meta-analysis using individual patient data from four randomised controlled trials. Thorax 2014; 69: 1128-1135

17. McEvoy RD, Antic NA, Heeley E, et al. CPAP for prevention of cardiovascular events in obstructive sleep apnea. N EnglJ Med 2016; 375: 919-931.
18. Yu J, Zhou Z, McEvoy RD, et al. Association of positive airway pressure with cardiovascular events and death in adults with sleep apnea: a systematic review and meta-analysis. JAMA 2017: 318: 156-166.

19. Nagayoshi M, Punjabi NM, Selvin E, et al. Obstructive sleep apnea and incident type 2 diabetes. Sleep Med 2016; 25 : 156-161.

20. Labarca G, Reyes T, Jorquera J, et al. CPAP in patients with obstructive sleep apnea and type 2 diabetes mellitus: systematic review and meta-analysis. Clin Respir J 2018; 12 : 2361-2368.

21. Gupta MA, Simpson FC, Lyons DC. The effect of treating obstructive sleep apnea with positive airway pressure on depression and other subjective symptoms: a systematic review and meta-analysis. Sleep Med Rev 2016; 28: 55-68.

22. Nanthakumar S, Bucks RS, Skinner TC. Are we overestimating the prevalence of depression in chronic illness using questionnaires? Meta-analytic evidence in obstructive sleep apnoea. Health Psychol 2016; 35: 423-432.

23. Stranks EK, Crowe SF. The cognitive effects of obstructive sleep apnea: an updated meta-analysis. Arch Clin Neuropsychol 2016; 31: 186-193.

24. Kylstra WA, Aaronson JA, Hofman WF, et al. Neuropsychological functioning after CPAP treatment in obstructive sleep apnea: a meta-analysis. Sleep Med Rev 2013; 17: 341-347.

25. Liguori C, Mercuri NB, Izzi F, et al. Obstructive sleep apnea is associated with early but possibly modifiable alzheimer's disease biomarkers changes. Sleep 2017; 40: zsx011.

26. Troussiere AC, Charley CM, Salleron J, et al. Treatment of sleep apnoea syndrome decreases cognitive decline in patients with Alzheimer's disease. J Neurol Neurosurg Psychiatry 2014; 85: 1405-1408 